\title{
Medicinal Plants Used by the Inhabitants of Alfred Nzo District Municipality in the Eastern Cape Province, South Africa
}

\author{
Zingisa Thinyane and Alfred Maroyi
}

\author{
Medicinal Plants and Economic Development (MPED) Research Centre, Department of Botany, University of \\ Fort Hare, Private Bag X1314, Alice 5700, South Africa
}

\begin{abstract}
Plant species used as herbal medicines play an important in the provision of primary healthcare in several rural communities. The current study was aimed at documenting medicinal plants used by the inhabitants of Alfred Nzo District Municipality in the Eastern Cape province, South Africa. Information on medicinal plants used for primary healthcare was collected through open-ended interviews with a sample of 124 participants selected via snowballsampling technique between April 2017 and May 2018. A total of 34 plant species and one fungus species representing 20 families were used in the treatment of 13 different human diseases. The major diseases treated by the documented species included respiratory system, pain, sores and wounds, infections and infestations, digestive system, blood and cardiovascular system, fever and malaria, general ailments, reproductive system and sexual health and mental disorders. Popular herbal medicines with relative frequency citation (RFC) values exceeding 0.50 included Bulbine frutescens, Clivia miniata var. miniata, Elephantorrhiza elephantina, Centella asiatica, Hypoxis hemerocallidea, Dicerothamnus rhinocerotis, Leonotis leonurus, Agapanthus africanus and Datura stramonium. Such repository of medicinal plants and fungi reinforces the need for an evaluation of their biological activities as a basis for developing future medicines and pharmaceutical products.
\end{abstract}

Keywords: Alfred Nzo District Municipality, fungi, herbal medicines, South Africa, traditional knowledge.

\section{INTRODUCTION}

Several pharmaceutical drugs and health promoting products have been extracted from plant species. Van Wyk et al. [1] argued that plant species were once a primary source of all the medicines in the world and they still continue to provide mankind with new remedies. Natural products derived from plants and their derivatives represent more than $50 \%$ of all pharmaceutical drugs in clinical use in the world with higher plants contributing no less than $25 \%$ of the total [2]. Well-known examples of plant derived medicines include quinine (Cinchona pubescens Vahl), morphine (Papaver somniferum L.), codeine (Papaver bracteatum Lindl.), salicylic acid (Salix sp.), curare (Chondrodendron tomentosum Ruiz \& Pavón), digitoxin (Digitalis purpurea L.), taxol (Taxus brevifolia Nutt.), atropine (Atropa belladonna L.), reserpine (Rauwolfia vomitoria Afzel) and vincristine (Catharanthus roseus (L.) G. Don) [1]. These discoveries generated a lot of interest in natural plant-based remedies as a source for pharmaceutical drugs and health promoting products.

In South Africa, medicinal plants are an important aspect of the daily lives of many people and they are an important part of the local people's cultural heritage [1]. Street and Prinsloo [3] argued that South Africa as

*Address correspondence to this author at the Medicinal Plants and Economic Development (MPED) Research Centre, Department of Botany, University of Fort Hare, Private Bag X1314, Alice 5700, South Africa; Tel: 0027719600326; Fax: 0027866177642; E-mail: amaroyi@ufh.ac.za

ISSN: 2223-3806 / E-ISSN: 1927-5951/19 a country is known for a strong history of traditional healing, hosts a variety of around 30000 flowering plant species and over 3400 plant species in southern Africa are used for medicinal purposes [4,5]. Street and Prinsloo [3] also argued that medicinal and aromatic plants are now universally recognised as the basis for a number of critical human health, social and economic support systems and benefits. Similarly, Mander et al. [6] argued that for many people in South Africa, traditional medicine is not considered an inferior alternative to western medicine, but is thought to be desirable and necessary for treating a range of health problems that western medicine does not treat adequately. These authors argued that $72 \%$ of the Black African population in South Africa from a diverse range of age categories, education levels, religions and occupations are known to use traditional medicines. Therefore, use of traditional medicines in South Africa is by choice and not a result of access and cost issues associated with western medicines. Although, various ethnobotanical surveys have been conducted in different parts of South Africa, some parts of the Eastern Cape province are still poorly explored. The Eastern Cape province is characterized by low socioeconomic standing with predominantly rural population suggesting that the majority of people in the province use traditional methods of healthcare [7-9]. It is therefore, within this context that the present study was undertaken aimed at providing the inventory of medicinal plant species used by the inhabitants of

(c) 2019 SET Publisher 
Alfred Nzo District Municipality in the Eastern Cape province, South Africa.

\section{MATERIALS AND METHODS}

\section{Description of the Study Area}

The study was conducted in Alfred Nzo District Municipality in the Eastern Cape Province, South Africa (Figure 1). The research study focused on three local municipalities, that is, Mbizana, Mzimvubu and Ntabankulu Local Municipalities (Figure 1). Alfred Nzo District Municipality is located between longitude $29^{\circ} 20^{\prime}$ $E$ to $30^{\circ} 10^{\prime} \mathrm{E}$ and latitude $30^{\circ} 60^{\prime} \mathrm{S}$ to $30^{\circ} 30^{\prime} \mathrm{S}$ at an altitude ranging from 0 to $1055 \mathrm{~m}$ above sea level ${ }^{11}$. The temperature varies from $7^{\circ} \mathrm{C}$ to $10^{\circ} \mathrm{C}$ in cool dry season, when it can also be snowy and from $18^{\circ} \mathrm{C}$ to $24^{\circ} \mathrm{C}$ in the hot wet season. The rainfall season is between October and March with the latter being the wettest month and rainfall varies from $750 \mathrm{~mm}$ to 1050 $\mathrm{mm}$ per annum [10]. Mucina and Rutherford [11] described the dominant vegetation of Alfred Nzo District Municipality as sub-escarpment grassland and sub-escarpment savanna bioregions dominated by moist grasslands and Acacia species.

The majority of communities in Alfred Nzo District Municipality are predominantly rural with the dominant land use practise being rearing of livestock and dryland crop production. The residents raise goats together with other livestock species such as cattle, sheep, chickens and pigs [10]. The arable lands are typically consolidated rain fed farming areas. With high levels of poverty, low levels of economic activity and the poor quality of land allocated to households in Alfred Nzo District Municipality, non-farm activities are potentially an important source of livelihood for the residents. Local people rely primarily on river and rainwater, while fuelwood and paraffin constitute the primary energy sources [12]. Major crops cultivated in the study area include maize (Zea mays L.), potatoes (Solanum tuberosum L.), cabbage (Brassica oleracea L.), spinach (Spinacia oleracea L.), beetroot (Beta vulgaris L.) and carrots (Daucas carota L.). The majority of the inhabitants (at least 87\%) in the study sites are traditional isiXhosa speaking people who are highly

\section{STUDY AREA}

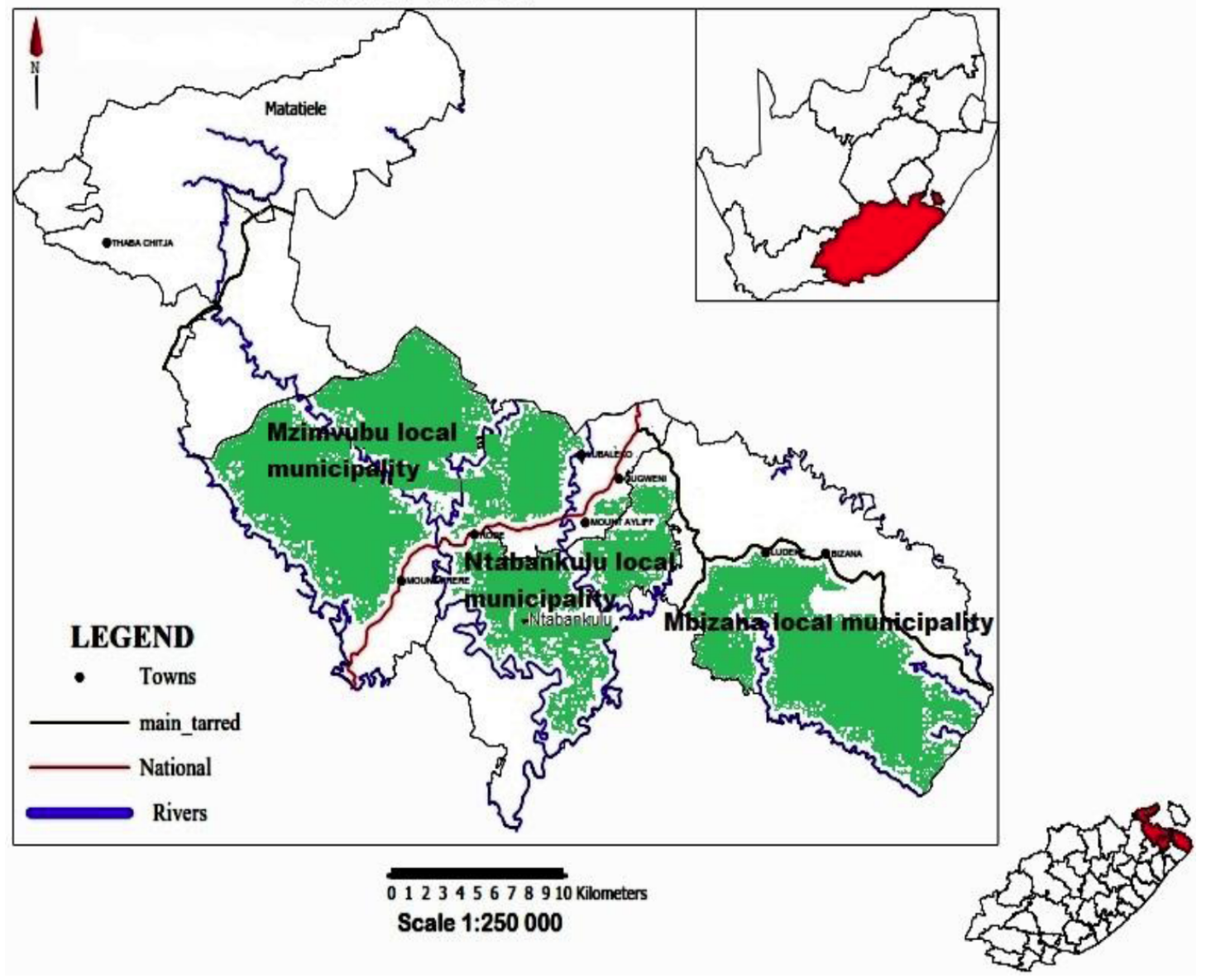

Figure 1: Map of South Africa showing the geographical position of the study areas in Alfred Nzo District Municipality. 
dependent on natural resources for their livelihood needs $[8,9]$.

\section{Data Collection}

The study was conducted between April 2017 and May 2018 documenting medicinal uses of plant species in the study area via interviews with 124 participants. Two data collection techniques, namely, snowball or chain-referral-sampling $[13,14]$ and participatory rural appraisal (PRA) [15,16] techniques were used. Snowball sampling or chain-referral-sampling of a study sample begins with a convenience sample of initial subject, and this initial subject serve as "seeds," through which wave 1 subject is recruited; wave 1 subject in turn recruit wave 2 subjects; and the sample consequently expands wave by wave like a snowball growing in size as it rolls down a hill $[13,14]$. Etikan et al. [14] argued that snowball sampling generates biased samples because respondents who have great number of social connections are able to provide investigators with a higher proportion of other respondents who have characteristics similar to that of initial respondents. Fundamental to PRA is the use of hands-on methods, such as participatory mapping and diagramming resources flows, which are thought to overcome communication barriers that may exist between outside researchers and community members [16]. Similarly, Cornwall and Pratt [17] described PRA as a family of approaches and methods to enable rural people to share, enhance and analyze their knowledge of life and conditions, to plan and to act. Structured and semi-structured interviews were carried out in isiXhosa, a language spoken by all participants. In order to ensure that participant's right to voluntarily decide to participate in this research on medicinal plants. All participants were encouraged to sign a consent form, after the researcher or research assistants had fully explained the nature of research work and acknowledged indigenous prior rights of the participants. The researchers also agreed to a working relationship with the community, including willingness to comply with indigenous or local and cultural governance systems, customs, laws and protocols as stipulated by the International Society of Ethnobiology (www.ethnobiology.net). The medicinal plants were initially identified by participants using their vernacular names, Xhosa names. The voucher specimens of medicinal plant species with fruits, leaves, pods, flowers were collected and processed using standard taxonomic procedures $[18,19]$ and incorporated into the Giffen Herbarium, University of Fort Hare as reference material.

\section{Data Analysis}

The collected data were carefully reviewed and assessed for accuracy, credibility, validation and reliability by the researcher prior to the analyses. The data were then analysed based on the information received from the local respondents using descriptive statistics such as frequencies, Microsoft Excel and Statistical Package for the Social Sciences (SPSS). We determined the Relative Frequency of Citation (RFC) of reported plant species using the following formula:

$$
\mathrm{RFC}=\mathrm{FC} / \mathrm{N} ;(0<\mathrm{RFC}<1)
$$

This index shows the local importance of each species and is given by the frequency of citation (FC), that is the number of informants mentioning the use of species divided by the total number of informants participating in the study $[20,21]$.

\section{RESULTS}

\section{Demographic and Socio-Economic Characteristics of the Participants}

A total of 124 participants selected via snowball technique from different local communities in Alfred Nzo District Municipality took part in this study. Table 1 provides demographic and socio-economic characteristics of the participants who provided information on herbal medicines in Alfred Nzo District Municipality. The majority of the participants $(57.3 \%)$ were males while $42.7 \%$ were females (Table 1). Close to three quarters of the participants $(70.2 \%)$ were between 26 years to 65 years, while $12.9 \%$ and $16.9 \%$ were below 26 years and above 66 years of age, respectively (Table 1). About a quarter of the participants $(25.0 \%)$ were illiterate, while $18.6 \%$ of the participants were educated up to primary level, $37.2 \%$ of the sample were educated up to secondary level and $19.4 \%$ of the sample had attained tertiary education (Table 1). The majority of families $(87.1 \%$ of households) comprised between four and more than ten household members (Table 1). More than half of the sample $(50.8 \%$ of the participants) were married, $25.8 \%$ were single, $13.7 \%$ and $9.7 \%$ were widowed and divorced, respectively (Table 1). The majority of the participants $(30.6 \%$ and $21.8 \%)$ were employed by government departments and private sector, respectively, while $20.2 \%$ had retired, $15.3 \%$ selfemployed and $12.1 \%$ were unemployed (Table 1). About a third of the participants $(29.8 \%)$ earned less than R2000.00 per month while about a quarter of the participants (21.8\%) earned more than R10001.00 (Table 1). 
Table 1: Demographic and Socio-Economic Characteristics of the Participants who Provided Information on Medicinal Plants in Alfred Nzo District Municipality, N = 124

\begin{tabular}{|c|c|c|}
\hline Characteristics & No. of informants & Percentage (\%) \\
\hline \multicolumn{3}{|l|}{ Gender } \\
\hline Females & 53 & 42.7 \\
\hline \multicolumn{3}{|l|}{ Age } \\
\hline $26-35$ years & 24 & 19.4 \\
\hline $36-45$ years & 25 & 20.2 \\
\hline $46-55$ years & 20 & 16.1 \\
\hline $56-65$ years & 18 & 14.5 \\
\hline Primary & 23 & 18.6 \\
\hline Secondary & 46 & 37.2 \\
\hline Tertiary & 24 & 19.4 \\
\hline \multicolumn{3}{|l|}{ Household size } \\
\hline $1-3$ members & 16 & 12.9 \\
\hline 4-6 members & 34 & 27.4 \\
\hline 7-9 members & 45 & 36.3 \\
\hline$>10$ members & 29 & 23.4 \\
\hline \multicolumn{3}{|l|}{ Occupation } \\
\hline Employed by government & 38 & 30.6 \\
\hline Employed by private sector & 27 & 21.8 \\
\hline Self-employed & 19 & 15.3 \\
\hline Unemployed & 15 & 12.1 \\
\hline Retired & 25 & 20.2 \\
\hline \multicolumn{3}{|l|}{ Income per month } \\
\hline$<\mathrm{R} 2000^{*}$ & 37 & 29.8 \\
\hline R2001 - 4000 & 13 & 10.5 \\
\hline R4001 - 6000 & 11 & 8.9 \\
\hline R6001 - 8000 & 12 & 9.7 \\
\hline R8001 - 10000 & 20 & 16.1 \\
\hline > R10001 & 27 & 21.8 \\
\hline Not disclosed & 4 & 3.2 \\
\hline
\end{tabular}

*14.00 South Africa Rand (R) $=1.00$ US $\$$. 
Table 2: List of Medicinal Plants Used in Alfred Nzo District Municipality. Species Marked with an Asterisk (*) are Exotic to South Africa

\begin{tabular}{|c|c|c|c|c|c|}
\hline Scientific and family names & Local name & Habit & Part s used & Species uses & RFC \\
\hline $\begin{array}{l}\text { *Datura stramonium L., } \\
\text { Solanaceae }\end{array}$ & $\begin{array}{l}\text { ljoyi and } \\
\text { umhlabavuthwa }\end{array}$ & Shrub & $\begin{array}{l}\text { Leaves and } \\
\text { roots }\end{array}$ & $\begin{array}{l}\text { Decoction taken orally as aphrodisiac, against } \\
\text { asthma, respiratory difficulties, tonsils, bronchitis, } \\
\text { sore throat, pain and gout, and applied topically } \\
\text { against rheumatism, boils, wounds and toothache }\end{array}$ & 0.94 \\
\hline $\begin{array}{l}\text { Agapanthus africanus (L.) } \\
\text { Hoffmanns, Agapanthaceae }\end{array}$ & Isicakathi & Herb & $\begin{array}{l}\text { Leaves and } \\
\text { roots }\end{array}$ & $\begin{array}{c}\text { Infusion taken orally against headache and to } \\
\text { induced labour }\end{array}$ & 0.85 \\
\hline $\begin{array}{c}\text { Leonotis leonurus (L.) R. Br., } \\
\text { Lamiaceae }\end{array}$ & Imficamficane & Shrub & $\begin{array}{l}\text { Leaves and } \\
\text { roots }\end{array}$ & $\begin{array}{l}\text { Decoction taken orally against coughs, asthma, } \\
\text { headache, common colds and tapeworms, and } \\
\text { applied topically against snake bites }\end{array}$ & 0.75 \\
\hline $\begin{array}{l}\text { Dicerothamnus rhinocerotis } \\
\text { (L.f.) Koekemoer, Asteraceae }\end{array}$ & Umlingatho & Shrub & $\begin{array}{l}\text { Leaves and } \\
\text { roots }\end{array}$ & $\begin{array}{l}\text { Decoction taken orally to improve appetite, and } \\
\text { against ulcers, dyspepsia and stomach cancer }\end{array}$ & 0.69 \\
\hline $\begin{array}{l}\text { Hypoxis hemerocallidea } \\
\text { Fisch., C.A. Mey \& Avé-Lall, } \\
\text { Hypoxidaceae }\end{array}$ & Inkomfe & Herb & $\begin{array}{l}\text { Corms, } \\
\text { leaves and } \\
\text { roots }\end{array}$ & $\begin{array}{l}\text { Decoction taken orally against urinary tract } \\
\text { infections, rheumatoid arthritis, tuberculosis, } \\
\text { cancer, testicular tumours, depression and anxiety }\end{array}$ & 0.67 \\
\hline $\begin{array}{l}\text { Bulbine latifolia (L.f.) Spreng., } \\
\text { Asphodelaceae }\end{array}$ & $\begin{array}{l}\text { Incelwani, } \\
\text { Ibhucu }\end{array}$ & Herb & $\begin{array}{l}\text { Leaf sap } \\
\text { and roots }\end{array}$ & $\begin{array}{c}\text { Decoction taken orally against diarrhoea, } \\
\text { dysentery, vomiting and diabetes, and applied } \\
\text { topically against wound, burns, eczema, rashes } \\
\text { and rheumatism }\end{array}$ & 0.64 \\
\hline $\begin{array}{l}\text { Centella asiatica (L.) Urb., } \\
\text { Apiaceae }\end{array}$ & Udingu & Herb & $\begin{array}{l}\text { Leaves and } \\
\text { roots }\end{array}$ & $\begin{array}{c}\text { Decoction taken orally against influenza, common } \\
\text { colds, blood purification, diarrhoea, dysentery and } \\
\text { enhance memory, and also applied topically } \\
\text { against wounds }\end{array}$ & 0.58 \\
\hline $\begin{array}{l}\text { Elephantorrhiza elephantina } \\
\text { (Burch.) Skeels, Fabaceae }\end{array}$ & Intolwane & Shrub & $\begin{array}{l}\text { Leaves and } \\
\text { roots }\end{array}$ & $\begin{array}{l}\text { Decoction taken orally against diarrhoea, } \\
\text { dysentery, syphilis, bleeding and heart ailments, } \\
\text { and ointment used for sun burn }\end{array}$ & 0.56 \\
\hline $\begin{array}{l}\text { Clivia miniata (Lindl.) Regel } \\
\text { var. miniata, Amaryllidaceae }\end{array}$ & Umayime & Herb & $\begin{array}{l}\text { Leaves and } \\
\text { rhizomes }\end{array}$ & $\begin{array}{c}\text { Decoction taken orally against fever, pain, } \\
\text { parturition and assist in child birth, and applied } \\
\text { topically against snake bite }\end{array}$ & 0.52 \\
\hline $\begin{array}{l}\text { Bulbine frutescens (L.) Willd., } \\
\text { Asphodelaceae }\end{array}$ & Ibhucu & Herb & $\begin{array}{l}\text { Leaves and } \\
\text { roots }\end{array}$ & $\begin{array}{l}\text { Decoction applied topically on wounds, burns, } \\
\text { rashes, itches, ringworms, cracked lips and herpes }\end{array}$ & 0.51 \\
\hline $\begin{array}{l}\text { Helichrysum odoratissimum } \\
\text { (L) Sweet, Asteraceae }\end{array}$ & $\begin{array}{l}\text { Impepho and } \\
\text { isiqhumiso }\end{array}$ & Herb & $\begin{array}{l}\text { Leaves and } \\
\text { stems }\end{array}$ & $\begin{array}{c}\text { Decoction taken orally against fever, colds, coughs, } \\
\text { headache, febrile convulsions, insanity and pain, } \\
\text { and applied topically for muscle cramps and } \\
\text { wounds }\end{array}$ & 0.49 \\
\hline $\begin{array}{l}\text { Zantedeschia aethiopica (L.) } \\
\text { Spreng., Araceae }\end{array}$ & Inyiba & Herb & $\begin{array}{l}\text { Leaves and } \\
\text { stems }\end{array}$ & $\begin{array}{c}\text { Decoction applied topically on wounds, sores, boils, } \\
\text { rheumatism, and taken orally against gout, heart } \\
\text { burn and sore throat }\end{array}$ & 0.49 \\
\hline $\begin{array}{l}\text { Dicoma capensis Less, } \\
\text { Asteraceae }\end{array}$ & $\begin{array}{l}\text { Ucelezi and } \\
\text { umuna }\end{array}$ & Herb & $\begin{array}{l}\text { Leaves and } \\
\text { roots }\end{array}$ & $\begin{array}{l}\text { Decoction taken orally against fever, influenza, high } \\
\text { blood pressure, stomach problems and diarrhoea }\end{array}$ & 0.47 \\
\hline $\begin{array}{l}\text { *Solanum nigrum L., } \\
\text { Solanaceae }\end{array}$ & Umsobo & Herb & $\begin{array}{l}\text { Leaves and } \\
\text { stems }\end{array}$ & $\begin{array}{l}\text { Decoction taken orally as appetite stimulant, } \\
\text { against fever, asthma, ulcers, pain, tuberculosis, } \\
\text { dysentery and stomach complaints, and also } \\
\text { topically against skin disorders }\end{array}$ & 0.43 \\
\hline $\begin{array}{l}\text { Aloe ferox Mill., } \\
\text { Asphodelaceae }\end{array}$ & $\begin{array}{l}\text { Ikhala and } \\
\text { Umhlaba }\end{array}$ & Shrub & $\begin{array}{l}\text { Leaves and } \\
\text { leaf sap }\end{array}$ & $\begin{array}{l}\text { Decoction taken orally as laxative, against arthritis } \\
\text { and hypertension; and topically for skin problems, } \\
\text { wounds and conjunctivitis. }\end{array}$ & 0.40 \\
\hline $\begin{array}{l}\text { Rumex lanceolatus Thunb., } \\
\text { Polygonaceae }\end{array}$ & $\begin{array}{l}\text { Idololenkonyane } \\
\text { and Inkonyane }\end{array}$ & Herb & $\begin{array}{l}\text { Leaves and } \\
\text { stems }\end{array}$ & $\begin{array}{l}\text { Decoction taken orally against tapeworms, round } \\
\text { worms, tumours, and internal bleeding }\end{array}$ & 0.40 \\
\hline $\begin{array}{l}\text { Aloiampelos ciliaris (Haw.) } \\
\text { Klopper \& Gideon F. Sm. var } \\
\text { cilliaris, Asphodelaceae }\end{array}$ & Isihlungu & Herb & Leaves & $\begin{array}{l}\text { Decoction taken orally against constipation and } \\
\text { ulcers and topically against skin disorders }\end{array}$ & 0.39 \\
\hline $\begin{array}{l}\text { Alepidea amatymbica Ackl. \& } \\
\text { Zeyh., Apiaceae }\end{array}$ & lqwili & Herb & $\begin{array}{l}\text { Leaves, } \\
\text { rhizomes } \\
\text { and roots }\end{array}$ & $\begin{array}{l}\text { Infusion taken orally against colds, coughs, } \\
\text { rheumatism and applied topically on wounds }\end{array}$ & 0.37 \\
\hline
\end{tabular}


(Table 2). Continued.

\begin{tabular}{|c|c|c|c|c|c|}
\hline Scientific and family names & Local name & Habit & Part s used & Species uses & RFC \\
\hline $\begin{array}{l}\text { *Psidium guajava L., } \\
\text { Myrtaceae }\end{array}$ & Gwava & Tree & $\begin{array}{l}\text { Fruits and } \\
\text { leaves }\end{array}$ & $\begin{array}{c}\text { Decoction taken orally against fever, coughs, } \\
\text { ulcers, boils, wounds, malaria, diarrhoea and } \\
\text { diabetes }\end{array}$ & 0.36 \\
\hline $\begin{array}{l}\text { *Aloe vera (L.) Burm f., } \\
\text { Asphodelaceae }\end{array}$ & Intlaba & Herb & $\begin{array}{l}\text { Leaves, leaf } \\
\text { sap and } \\
\text { stems }\end{array}$ & $\begin{array}{l}\text { Decoction taken orally against constipation and } \\
\text { ulcers, and topically against skin disorders. }\end{array}$ & 0.31 \\
\hline $\begin{array}{c}\text { *Prunus persica (L.) Batsch, } \\
\text { Rosaceae }\end{array}$ & Ipesika & Tree & $\begin{array}{l}\text { Leaves and } \\
\text { stems }\end{array}$ & $\begin{array}{c}\text { Decoction taken orally against cough, whooping } \\
\text { cough, asthma, and applied topically on sores and } \\
\text { wounds }\end{array}$ & 0.31 \\
\hline $\begin{array}{l}\text { Typha capensis (Rohrb.) } \\
\text { N.E.Br., Typhaceae }\end{array}$ & Ingcongolo & Herb & $\begin{array}{l}\text { Leaves and } \\
\text { roots }\end{array}$ & $\begin{array}{l}\text { Infusion taken orally for fertility in women, } \\
\text { expansion of uterine during birth, improve blood } \\
\text { circulation, diarrhoea and dysentery }\end{array}$ & 0.30 \\
\hline $\begin{array}{l}\text { *Cannabis sativa L., } \\
\text { Cannabaceae }\end{array}$ & Somntsangu & Shrub & $\begin{array}{l}\text { Leaves and } \\
\text { twigs }\end{array}$ & $\begin{array}{l}\text { Smoked as a cigarette for cough, colds, fever, } \\
\text { headache and enhance memory }\end{array}$ & 0.28 \\
\hline $\begin{array}{l}\text { *Foeniculum vulgare Mill., } \\
\text { Apiaceae }\end{array}$ & Imboziso & Herb & Leaves & Decoction taken orally for stimulating appetite & 0.27 \\
\hline $\begin{array}{l}{ }^{*} \text { Catharanthus roseus (L.) G. } \\
\text { Don, Apocynaceae }\end{array}$ & Isihlungu & Shrub & $\begin{array}{l}\text { Leaves and } \\
\text { roots }\end{array}$ & $\begin{array}{l}\text { Infusion taken orally against cancer and diabetes } \\
\text { and applied topically against rheumatism }\end{array}$ & 0.26 \\
\hline $\begin{array}{l}\text { Aloe greatheadii Schönland } \\
\text { var. davyana. (Schönland) } \\
\text { Glen \& D.S. Hardy, } \\
\text { Asphodelaceae }\end{array}$ & Inkala & Herb & $\begin{array}{l}\text { Leaves and } \\
\text { leaf sap }\end{array}$ & $\begin{array}{l}\text { Decoction taken orally against cancer or applied } \\
\text { apically against wounds, sores, various skin } \\
\text { ailments such as eczema, skin irritation and bruises }\end{array}$ & 0.25 \\
\hline $\begin{array}{c}\text { Conyza scabrida DC., } \\
\text { Asteraceae }\end{array}$ & Isavu & Shrub & $\begin{array}{l}\text { Leaves and } \\
\text { twigs }\end{array}$ & $\begin{array}{c}\text { Decoction taken orally against stomach aches, } \\
\text { chest, heart problems, inflammation, coughs, colds, } \\
\text { flu, fever and diabetes, and applied topically } \\
\text { against rheumatism }\end{array}$ & 0.24 \\
\hline $\begin{array}{l}\text { Lippia javanica (Burm. f.) } \\
\text { Spreng., Verbenaceae }\end{array}$ & inzinziniba & Shrub & $\begin{array}{l}\text { Leaves and } \\
\text { stems }\end{array}$ & $\begin{array}{l}\text { Decoction taken orally against bronchial problems, } \\
\text { coughs, fever, malaria and lung problems }\end{array}$ & 0.23 \\
\hline $\begin{array}{c}\text { Artemisia afra Jacq. ex. Willd., } \\
\text { Asteraceae }\end{array}$ & Umhlonyane & Shrub & $\begin{array}{l}\text { Leaves and } \\
\text { stems }\end{array}$ & $\begin{array}{c}\text { Decoction taken orally against fever, cough, } \\
\text { respiratory problems, flue and stomach } \\
\text { complications }\end{array}$ & 0.22 \\
\hline $\begin{array}{l}\text { *Caesalpinia decapetala } \\
\text { (Roth) Alson, Fabaceae }\end{array}$ & Bobo & Shrub & $\begin{array}{l}\text { Fruits and } \\
\text { stems }\end{array}$ & $\begin{array}{l}\text { Decoction taken orally against dysentery, malaria } \\
\text { and pain. }\end{array}$ & 0.20 \\
\hline $\begin{array}{l}\text { Tecoma capensis (Thunb.) } \\
\text { Lindl., Bignoniaceae }\end{array}$ & Umsilingi & Shrub & $\begin{array}{l}\text { Leaves and } \\
\text { stems }\end{array}$ & $\begin{array}{l}\text { Decoction taken orally against bronchitis, stomach } \\
\text { pains, diarrhoea and dysentery }\end{array}$ & 0.19 \\
\hline $\begin{array}{l}\text { Helichrysum pedunculatum } \\
\text { Hilliard \& B.L. Burtt, } \\
\text { Asteraceae }\end{array}$ & Impepho & Herb & $\begin{array}{l}\text { Leaves and } \\
\text { stems }\end{array}$ & $\begin{array}{l}\text { Infusion taken orally against fever, respiratory } \\
\text { problems and lung problems }\end{array}$ & 0.15 \\
\hline $\begin{array}{l}\text { *Malva parviflora L. var. } \\
\text { parviflora, Malvaceae }\end{array}$ & Ujongelana & Herb & $\begin{array}{l}\text { Leaves and } \\
\text { roots }\end{array}$ & $\begin{array}{l}\text { Decoction taken orally against coughs and ulcers, } \\
\text { and applied topically on sores and wounds }\end{array}$ & 0.14 \\
\hline $\begin{array}{c}\text { Agaricus subrufescens Peck, } \\
\text { Agaricaceae }\end{array}$ & Ikhowa & Fungus & $\begin{array}{l}\text { Fruiting } \\
\text { bodies }\end{array}$ & Infusion taken orally against ovarian cancer tumour & 0.13 \\
\hline $\begin{array}{c}\text { Mentha longifolia (L.) L., } \\
\text { Lamiaceae }\end{array}$ & Inxina & Herb & $\begin{array}{l}\text { Leaves and } \\
\text { stems }\end{array}$ & $\begin{array}{c}\text { Infusion taken orally against respiratory problems, } \\
\text { cough, colds, stomach complications, asthma, } \\
\text { indigestion, menstrual pains and swollen glands, } \\
\text { and applied topically on wounds }\end{array}$ & 0.12 \\
\hline
\end{tabular}

\section{Diversity of Medicinal Plants}

This study recorded 34 plant and one mushroom species used traditionally to manage human diseases in Alfred Nzo District Municipality (Table 2). Of these, 25 species are indigenous to South Africa (71.4\%), while 10 species are exotic (28.6\%), either naturalized as weeds or cultivated in home gardens. The exotic species to South Africa included Aloe vera (L.) Burm. f., Caesalpinia decapetala (Roth) Alson, Cannabis sativa L., Catharanthus roseus (L.) G. Don, Datura stramonium L., Foeniculum vulgare Mill., Malva parviflora L. var. parviflora, Psidium guajava L. and Solanum nigrum L. These medicinal plants were distributed among 20 families and the majority of medicinal plants $(60.0 \%)$ were used in Alfred Nzo 
District Municipality (Table 3). Plant families with the highest number of medicinal plants in Alfred Nzo District Municipality were from six families: Asphodelaceae and Asteraceae (six species each), followed by Apiaceae (three species), Fabaceae, Lamiaceae and Solanaceae (two species each) (Table 3). Herbs (57.1\%) and shrubs (34.3\%) were the primary sources of herbal medicines in Alfred Nzo District Municipality (Figure 2A). The mushroom and plant parts used for herbal preparations were fruits, fruiting bodies, leaves, roots, stems, twigs and whole plant (Table 2). The leaves were the most frequently used $(88.6 \%)$, followed by stems $(34.3 \%)$, roots $(22.9 \%)$, fruits $(5.7 \%)$, fruiting bodies, twigs and whole plant (2.9\% each) (Figure 2B).

Table 3: Families with the Largest Number of Medicinal Plant Species in Alfred Nzo District Municipality

\begin{tabular}{|c|c|c|}
\hline Family & Number of species & Percentage \\
\hline \hline Asteraceae & 6 & 17.1 \\
\hline Asphodelaceae & 6 & 17.1 \\
\hline Apiaceae & 3 & 8.6 \\
\hline Fabaceae & 2 & 5.7 \\
\hline Lamiaceae & 2 & 5.7 \\
\hline Solanaceae & 2 & 5.7 \\
\hline
\end{tabular}

Popular medicinal plant with RFC values exceeding 0.50 included (in descending order of RFC values): Bulbine frutescens (L.) Willd., Clivia miniata (Lindl.) Regel var. miniata, Elephantorrhiza elephantina (Burch.) Skeels, Centella asiatica (L.) Urb., Hypoxis hemerocallidea Fisch., C.A. Mey \& Avé-Lall,
Dicerothamnus rhinocerotis (L.f.) Koekemoer, Leonotis leonurus (L.) R. Br., Agapanthus africanus (L.) Hoffmanns and Datura stramonium L. (Table 2). The medicinal applications of mushroom and plant species were classified into the following 13 medical categories following the Economic Botany Data Collection Standard [22] with some changes proposed by Macía et al. [23] and Gruca et al. [24]: blood and cardiovascular system, digestive system, fever and malaria, general ailments, infections and infestations, mental disorders, muscular-skeletal system disorders, pain, sores and wounds, pregnancy, birth and puerperium disorders, reproductive system and sexual health, respiratory system and urinary system (Table 2). The major diseases treated by the documented plant species included the following (in descending order of importance): respiratory system (22 citations), pain, sores and wounds (21 citations), infections and infestations (15 citations), digestive system (14 citations), blood and cardiovascular system (12 citations), fever and malaria (12 citations), general ailments (6 citations), reproductive system and sexual health (6 citations) and mental disorders (5 citations) (Figure 3).

\section{DISCUSSION}

\section{Demographic and Socio-Economic Characteristics of the Participants}

The average annual household income within the sampled community population was low as well as literacy levels with $43.6 \%$ either illiterate or educated up to primary level (Table 1) and this is probably due to limited education facilities within the study area. The current study showed that the majority of households
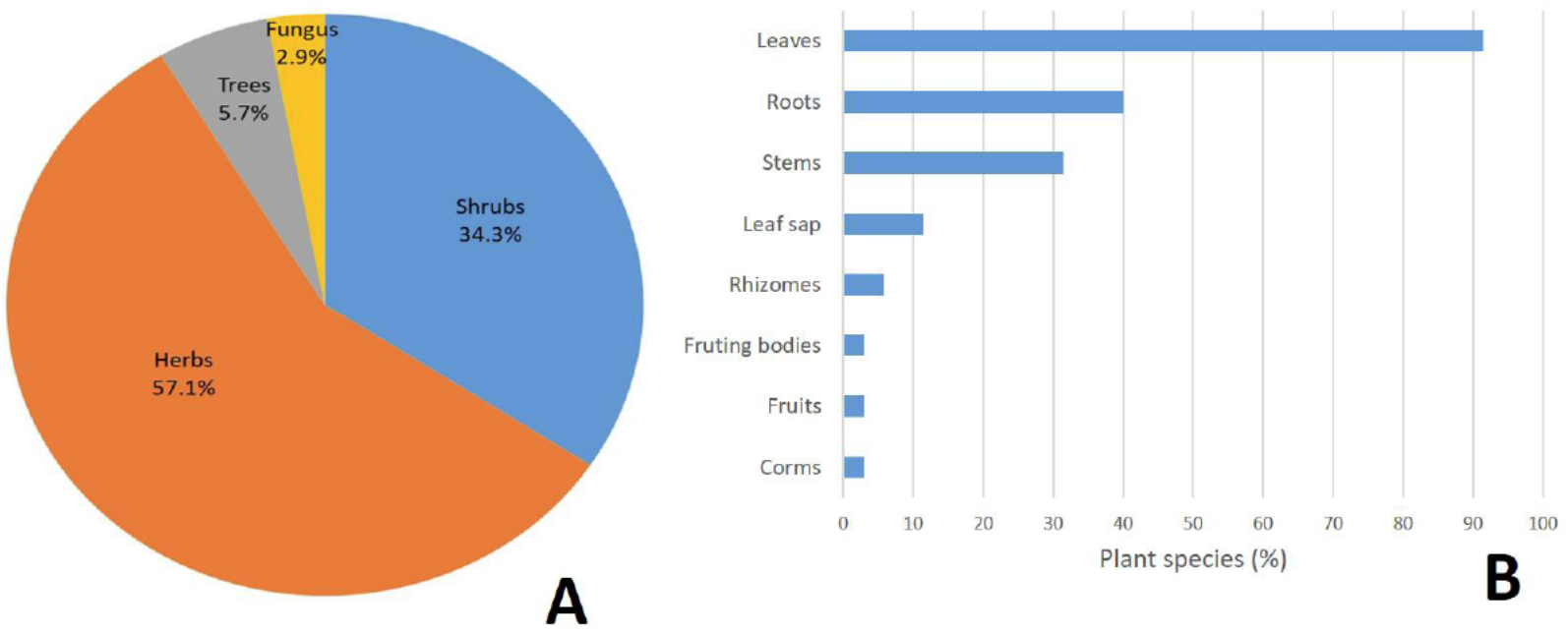

Figure 2: Characteristics of the plants and mushroom used as herbal medicines in Alfred Nzo District Municipality. (A) Growth form habit represented in pie diagram and (B) Fungus and plant parts used represented in bar chart. 


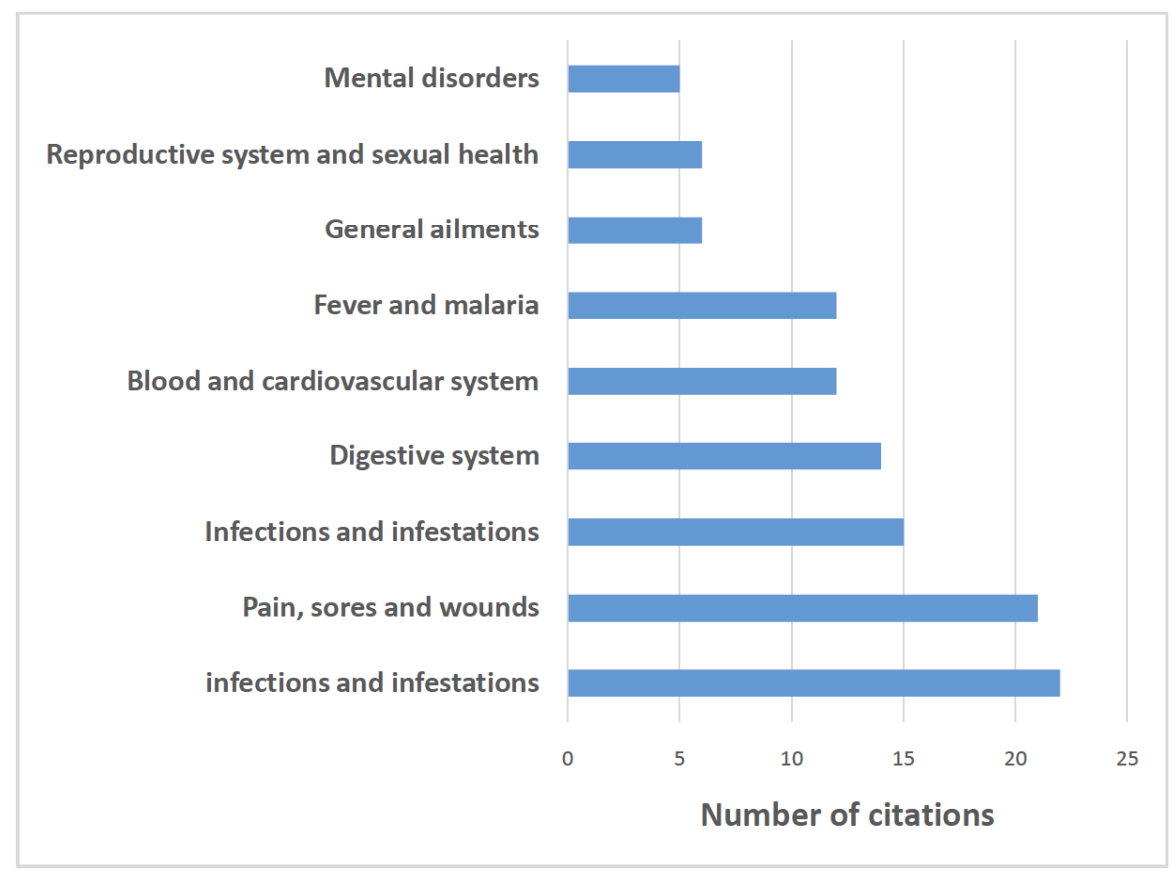

Figure 3: Major ailments and disease categories and number of plant species cited per category. Most species were reported in more than one ailment category.

are of low socio-economic standing, and therefore, these households use herbal medicines to fulfil their primary healthcare needs. Results of the current study correlate with research findings obtained by Paumgarten et al. [25] who revealed that the majority of the areas in the former homelands such as Ciskei and Transkei in the Eastern Cape Province are characterized by a strong reliance on migrant remittances and state pensions, low economic activity, low levels of education and poor skills base. Previous researchers, for example Dold and Cocks [7] and Maroyi [8,9] argued that since most areas in the Eastern Cape are predominantly rural, the large population in the province uses traditional methods of healthcare. Research by Maroyi [8] conducted in Ntabelanga dam catchment area, Eastern Cape province showed that out of 95 useful plant species, 46 species were used as herbal medicines. In another study conducted in six villages in the Eastern Cape province, Maroyi [9] found that the majority of the species $(62.4 \%)$ were used as herbal medicines while $18.4 \%$ were used as ethnoveterinary medicines. With a medical doctor to total population ratio in South Africa of 1:17 400 reported by Pretorius et al. [26] there is no doubt that traditional medicines play an important role in the nation's healthcare system. Campbell-Hall et al. [27] argued that traditional practitioners provide culturally appropriate care which is linked to indigenous explanatory models of illness held by many South Africans and such explanatory models of illness are understood to refer to how a person interprets their illness in relation to causation, precipitating events and initial symptoms, expected course of the illness as well as treatment options.

\section{Diversity of Medicinal Plants}

A significant number of medicinal plants $(28.6 \%)$ documented in Alfred Nzo District Municipality are exotic to South Africa and some of these including Caesalpinia decapetala, Catharanthus roseus, Datura stramonium and Psidium guajava pose an immediate and significant threat by virtue of their aggressive qualities and having the capacity to invade natural habitats and overwhelm some of the indigenous species [28-32]. Previous studies showed that invasive plants may also have positive economical, social and ecological significance and these need to be taken into account when assessing the costs resulting from invasions [33-41]. There is now a large body of evidence supporting human dependency on invasive alien plant species as herbal medicines [33-41]. The results of this study, therefore, calls for a review of the socio-economic benefits of exotic plants to local communities in South Africa before blindly advocating for their eradication. In addition, the extensive use of exotic plants is seen as imperative for their ultimate control and should ultimately form part of their management strategy [38].

Exotic plant species are now recognized as an important component of indigenous pharmacopoeia in 
several countries [42-46]. Alencar et al. [44] argued that any indigenous medical system is not a static social institution that is not evolving, as there is evidence of insertions and deletions of plants that compose it, with the addition of exotic plants as herbal medicines. In an earlier study, Alencar et al. [43] found that exotic plants are included in traditional pharmacopoeias to fill therapeutic vacancies that native plants cannot satisfy. While Palmer [47] argued that the medicinal plant collection of a community is the product of experimentations conducted throughout the history of a community and represents an adaptation of this culture over time. Bennett and Prance [42] argued that exotic plants that are used by a cultural group as food source or ornamental, may eventually be introduced into their traditional pharmacopoeias mainly because of the use-versatility of such species. Similarly, Alencar et al. [43] found exotic plants in Brazil to have more usecitations than native plants and the documented uses included applications as herbal medicines in addition to general uses as food plants or ornamentals.

\section{AUTHORS' CONTRIBUTIONS}

We declare that this work was done by the authors named in this article.

\section{CONFLICT OF INTEREST}

No conflict of interest is associated with this work.

\section{ACKNOWLEDGEMENTS}

The authors would like to express their gratitude to the National Research Foundation, South Africa (NRF) and Govan Mbeki Research and Development Centre (GMRDC), University of Fort Hare for financial support to conduct this study.

\section{REFERENCES}

[1] Van Wyk B-E, Oudtshoorn BV, Gericke N. Medicinal plants of South Africa. Pretoria: Briza Publications; 2013.

[2] Kinghorn AD, Balandrin MF. Human medicinal agents from plants. Washington DC: ACS Symposium Series 534, American Chemical Society; 1993.

[3] Street RA, Prinsloo G. 2013. Commercially important medicinal plants of South Africa: a review. J Chem 2013; vol 2013, Article ID 205048.

[4] Arnold TH, Prentice CA, et al. Medicinal and magical plants of southern Africa: an annotated checklist. Pretoria: Strelitzia 13. National Botanical Institute; 2002.

[5] Williams VL, Victor JE, Crouch NR. 2013. Red Listed medicinal plants of South Africa: status, trends, and assessment challenges. S Afr J Bot 2013; 86: 23-35. https://doi.org/10.1016/j.sajb.2013.01.006

[6] Mander $\mathrm{M}$, Ntuli $\mathrm{L}$, et al. Economics of the traditional medicine trade in South Africa. In South African health review, Harrison S, Bhana R, Ntuli A, Eds. Durban: Health Systems Trust; 2007, pp. 189-200.

[7] Dold AL, Cocks ML. The trade in medicinal plants in the Eastern Cape province, South Africa. S Afr J Sci 2002; 98: 589-597.

[8] Maroyi A. Assessment of useful plants in the catchment area of the proposed Ntabelanga dam in the Eastern Cape province, South Africa. The Sci World J 2017; vol 2017. Article ID 3763607. https://doi.org/10.1155/2017/3763607

[9] Maroyi A. Diversity of use and local knowledge of wild and cultivated plants in the Eastern Cape province, South Africa. J Ethnobiol Ethnomed 2017; 13: 43. https://doi.org/10.1186/s13002-017-0173-8

[10] Marume U, Chimonyo M, Dzama K. Some insights into the reproductive performance, selection criteria, production and market constraints in communal goat production systems of South Africa. Sci Res Essays 2013; 8(30): 1448-1458.

[11] Mucina L, Rutherford MC. The vegetation of South Africa Lesotho and Swaziland. Pretoria: Strelizia 19, South African National Biodiversity Institute; 2006.

[12] Paumgarten F, Shackleton CM. The role of non-timber forest products in household coping strategies in South Africa: the influence of household wealth and gender. Population Env 2011; 33(1): 108-131. https://doi.org/10.1007/s11111-011-0137-1

[13] Patton M. Qualitative evaluation and research methods. California: Sage Publications; 1990.

[14] Etikan I, Alkassim R, Abubakar S. Comparison of snowball sampling and sequential sampling technique. Biometrics Biostat Int J 2015; 3(1): 55.

[15] Chambers $R$. The origins and practice of participatory rural appraisal (PRA). World Develop 1994; 22: 953-969. https://doi.org/10.1016/0305-750X(94)90141-4

[16] Doyle R, Krasny M. Participatory rural appraisal as an approach to environmental education in urban community gardens. Env Ed Res 2003; 9(1): 91-115. https://doi.org/10.1080/13504620303464

[17] Cornwall A, Pratt G. The use and abuse of participatory rura appraisal: reflections from practice. Agr Human Values 2011 28(2): 263-272. https://doi.org/10.1007/s10460-010-9262-1

[18] Bridson D, Foreman L. The herbarium handbook. London: Royal Botanic Gardens, Kew, Richmond; 1998.

[19] Victor JE, Koekemoer $M$, et al. Herbarium essentials: the southern African herbarium user manual. Pretoria: SABONET Report no. 25. South African National Biodiversity Institute; 2004

[20] Tardío J, Pardo-de-Santayana M. Cultural importance indices: a comparative analysis based on the useful wild plants of Southern Cantabria (Northern Spain). Econ Bot 2008; 62: 24-39. https://doi.org/10.1007/s12231-007-9004-5

[21] Vitalini S, Tomè F, Fico G. Traditional uses of medicinal plants in Valvestino (Italy). J Ethnopharmacol 2009; 121: 106-116. https://doi.org/10.1016/j.jep.2008.10.005

[22] Cook FEM. Economic botany data collection standard. London: Prepared for the International Working Group on Taxonomic Databases for Plant Sciences (TDWG), Kew Royal Botanic Gardens, Kew; 1995.

[23] Macía MJ, Armesilla PJ, et al. Palm uses in northwestern South America: a quantitative review. The Bot Rev 2011; 77(4): 462-570. https://doi.org/10.1007/s12229-011-9086-8

[24] Gruca $M$, Cámara-Leret $R$, et al. New categories for traditional medicine in the Economic Botany Data Collection Standard. J Ethnopharmacol 2014; 155(2): 1388-1392. https://doi.org/10.1016/j.jep.2014.06.047 
[25] Paumgarten F, Shackleton CM, Cocks ML. Growing of trees in home gardens by rural households in the Eastern Cape and Limpopo provinces, South Africa. Int J Sust Develop World Ecol 2005; 12: 1-19.

https://doi.org/10.1080/13504500509469647

[26] Pretorius E., De Klerk G.W. and Van Rensburg H.C.J. 1993. The traditional healer in South African health care. Cooperative HSRC Programme, Affordable Social Provision Report ASS/BBS-27. Pretoria.

[27] Campbell-Hall V, Petersen I, et al. Collaboration between traditional practitioners and primary health care staff in South Africa: developing a workable partnership for community mental health services. Transcult Psych 2010; 47(4): 610628. https://doi.org/10.1177/1363461510383459

[28] South Africa. Conservation of agricultural resources act No. 43 of 1983. Pretoria: Government Printer; 1983.

[29] Henderson L. Alien weeds and invasive plants: a complete guide to declared weeds and invaders in South Africa including another 36 species invasive in that region. Pretoria: Plant Protection Research Institute Handbook No. 12. Agricultural Research Council; 2001.

[30] Henderson L. Invasive, naturalized and casual alien plants in southern Africa: a summary based on the southern African plant invaders atlas (SAPIA). Bothalia 2007; 37: 215-248. https://doi.org/10.4102/abc.v37i2.322

[31] Glen H, Van Wyk B. Guide to trees introduced into southern Africa. Cape Town: Struik Nature; 2016.

[32] Henderson L, Wilson JRU. Changes in the composition and distribution of alien plants in South Africa: an update from the southern African plant invaders atlas. Bothalia 2017; 47(2): a2172. https://doi.org/10.4102/abc.v47i2.2172

[33] Zimmermann HG, Neser S. Trends and prospects for biological control of weeds in South Africa. African Entomol Mem 1999; 1: 165-173.

[34] Stepp JR, Moerman DL. The importance of weeds in ethnopharmacology. J Ethnopharmacol 2001; 75: 19-23. https://doi.org/10.1016/S0378-8741(00)00385-8

[35] Njoroge NG, Bussmann WR, et al. Utilisation of weed species as sources of traditional medicines in central Kenya. Lyonia 2004; 7: 71-87.

[36] Bigirimana J, Bogaert J, et al. armentier I. Alien plant species dominate the vegetation in a city of sub-Saharan Africa. Landscape Urban Plan 2011; 100: 251-267. https://doi.org/10.1016/j.landurbplan.2010.12.012
[37] Bigirimana $\mathrm{J}$, Bogaert $\mathrm{J}$, et al. Domestic garden plant diversity in Bujumbura, Burundi: role of the socio-economical status of the neighbourhood and alien species invasion risk. Landscape Urban Plan 2012; 107: 118-126.

https://doi.org/10.1016/j.landurbplan.2012.05.008

[38] Semenya SS, Potgieter MJ, et al. Medicinal utilization of exotic plants by Bapedi traditional healers to treat human ailments in Limpopo Province, South Africa. J Ethnopharmacol 2012; 144: 646-655.

https://doi.org/10.1016/j.jep.2012.10.005

[39] Maroyi A. Use of weeds as traditional vegetables in Shurugwi district, Zimbabwe. J Ethnobiol Ethnomed 2013; 9: 60. https://doi.org/10.1186/1746-4269-9-60

[40] Maroyi A. Use and management of homegarden plants in Zvishavane district, Zimbabwe. Trop Ecol 2013; 54: 191-203.

[41] Semenya SS, Potgieter MJ. Bapedi traditional healers in the Limpopo province, South Africa: Their socio-cultural profile and traditional healing practice. J Ethnobiol Ethnomed 2014; 10: 4.

https://doi.org/10.1186/1746-4269-10-4

[42] Bennett BC, Prance GT. Introduced plants in the indigenous pharmacopoeia of northern South America. Econ Bot 2000; 54: 90-102. https://doi.org/10.1007/BF02866603

[43] Alencar NL, de Sousa TAA, et al. The inclusion and selection of medicinal plants in traditional pharmacopoeias-evidence in support of the diversification hypothesis. Econ Bot 2010; 64: 68-79.

https://doi.org/10.1007/s12231-009-9104-5

[44] Alencar NL, Santoro FR, Albuquerque UP. What is the role of exotic medicinal plants in local medical systems? A study from the perspective of utilitarian redundancy. Revista Brasil Farmacog 2014; 24: 506-515.

https://doi.org/10.1016/j.bjp.2014.09.003

[45] Maroyi A. Exotic plants in indigenous pharmacopoeia of south-central Zimbabwe: Traditional knowledge of herbal medicines. Res J Bot 2017; 12: 46-52. https://doi.org/10.3923/rib.2017.46.52

[46] Semenya SS, Maroyi A. Exotics plants used therapeutically by Bapedi traditional healers for respiratory infections and related symptoms in the Limpopo province, South Africa. Indian J Trad Knowl 2018; 17: 663-671.

[47] Palmer CT. The inclusion of recently introduced plants in the Hawaiian ethnopharmacopoeia. Econ Bot 2004; 58: S280S293.

https://doi.org/10.1663/00130001(2004)58[S280:TIORIP]2.0.CO;2 